\title{
VIRTUAL MODEL OF THE TERRAIN - A REALITY AVAILABLE TO EVERYONE
}

\author{
Nicolae MORO, Florin ILIE \\ "Nicolae Bălcescu" Land Forces Academy, Sibiu, Romania \\ nicu.moro@yahoo.com, ilieflorinv@yahoo.com
}

\begin{abstract}
Planning warfare at the tactical level, establishing and choosing the best course of action involves among other things, a precise knowledge of the configuration of the field in the area of engagement, disposition natural and artificial planimetric elements (layout) and their influence on warfare. Classical sandbox or sand table, tree branches, stones and other materials will be replaced with land simulation methods which located in the virtual sphere with minimal hardware and software investment and which will generate a report outstanding results with extremely high efficiency.
\end{abstract}

Keywords: terrain, analysis, virtual, sandbox.

\section{Introduction}

The complex, dynamic and permanent process of integrated analysis of the enemy, terrain, and ground confrontation environment within the operational environment is termed generic Intelligence preparation of battlefield.

As is well known by the military, the implementation algorithm of this type of analysis contains four mandatory steps, stages that cannot be circumvented or neglected in any form, each having its importance and role in substantiating the commander's decisions about choosing a optimum military course of action.

From the point of view of the subject approached in this paper it is appropriate to stop at the second stage, which highlights the terrestrial environment in its complexity and specificity and its influence on the operations. This land-based assessment always includes a detailed land-based analysis indissolubly by an analysis of the weather, season and weather conditions grafted on the other components of the operational environment, the environment as a whole described as a sum of variables that can influence the actions military.

Military conflicts at all times have underlined the need for a thorough and well-grounded analysis of the ground from a military point of view. The study of the land mainly concerns: the general study of the terrain character, the detailed study of the land aspect, the study of the observation and firing conditions, the covering and masking conditions, the sheltering and the protection, the study of the accessibility conditions of the land and very important, the examination of the obstacles and their influence on future intentions.

The best way invariably used by militaries in all armies of the world for tens and perhaps hundreds of years is to analyze the land at the so-called sandbox or sand case. It is an analysis that involves the one who performs a particularly logistic effort but also to a certain extent and physically to accurately represent the position and size of the various relief details and especially details of natural and artificial planimetry in the area to take place of the prefigured action. 
It is very important for this type of investigation that the fidelity and accuracy of the images provided does not affect the correct understanding and cognitive representation of the terrain configuration and the exact arrangement of all the existing planimetry details.

The objective of these research is to help these analysts and to provide them with a better and more accurate picture of the real terrain, but also a simpler, more representative and at the same time virtual way of performing this type of analysis. The result of these concerns was to design a virtual sandbox that would offer all these advantages that will result in a more objective analysis of the pitch at the tactical level.

\section{Materials}

The realization of this project involves the use of unusual materials for a classical sandbox because it combines hardware and software that together put together will be the source of this ground analysis support. This project will contain about $100 \mathrm{~kg}$ of quartz sand, a Kinect hardware platform, a video projector, a SFF (Small Form Factor) computer center unit, or a laptop, a wooden case that puts it into a single unit and the associate software.

The fine quartz sand, known as sand for filtering, is used for filtration and purification of water, has a decorative white color with uniformly distributed granulation (grain size) between $0.06-0.35 \mathrm{~mm}$, a hardness of 7 on a Mosh scale, with an uncompacted weight of $1100 \mathrm{~kg} / \mathrm{m}^{3}$, compacted weight of $1530 \mathrm{~kg} / \mathrm{m}^{3}$ and a specific density of $2636 \mathrm{~kg} / \mathrm{m}^{3}$. It is usually packaged and can be purchased in $25 \mathrm{~kg}$ bags, it is $100 \%$ safe for health, washed and selected, it does not form litometeors in air so it can not be inhaled during modeling, having a humidity of $1-3 \%$, which gives it a very important feature, that is a very good plasticity.

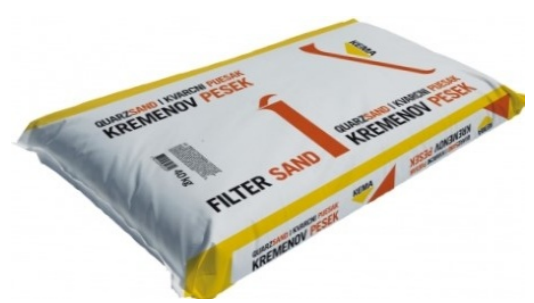

Figure 1 Quartz sandbag [1]

A perhaps more expensive variant and, in our opinion, not much more efficient is the kinetic sand, which in principle has a composition similar to fine quartz sand but is combined with a $2 \%$ special silicone oil (technical name of polydimethysiloxane) which it gives a higher degree of plasticity, hence a better remanence in time. This is not to be neglected because it is sometimes necessary for this sandbox to be transported and used in the field and integrity during transport is essential.

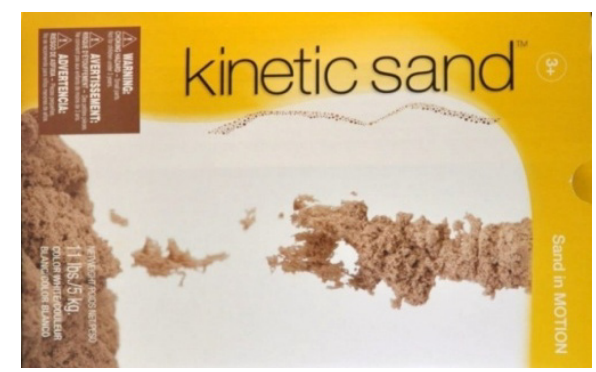

Figure 2 Kinetic sand 
A basic element of the panel is the Kinect hardware platform, which is a motion sensor and which aims to anticipate and take over the movements and changes in height the sandy surface receives by shaping it with the operator's hands. This kinect hardware uses a three-camera system, one with a resolution of $480 \times 640$ pixels that shoots the surface of the sand and views it on the optional monitor screen. The second lens is an infrared camera that scans the surface of the sand and detects variations in the height and shape of the sand surface. The most important is the third camera that functions as a very sensitive and accurate telemetry because it measures the distance traveled by the infrared ray from the lens to the surface of the sand. This fact compulsoriness the need to calibrate the whole system before starting the terrain analysis to determine the zero altitude reference value.

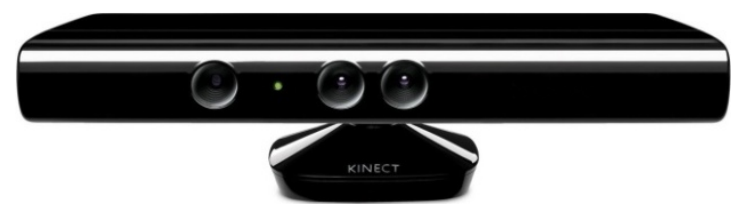

Figure 3 Kinetic motion sensor [2]

Essential for the quality of this experiment is the video spotlight used to transpose the required image over the surface of the sand. It is recommended that this hardware element displays the image in rich colors (at least 1 billion colors) and renders a high definition native resolution $(1920 \times 1080$ pixels) and a contrast of at least 10,000: 1 . If quartz sand is used then the luminous flux can have a minimum of 1000 lumens because the white of this sand will compensate for the lower luminous flux of the device. If kinetic sand is used then its brown color will require a stronger light output, estimated at a minimum of 3000 lumens. For a more realistic picture, it is advisable that the image is $16: 9$ (4:3), the video spotlight has a minimum image focus (distance to image) of $0.50 \mathrm{~m}$ (short throw video projector) and the minimum diagonal of the image formed to be at least as diagonal of the sand case for to have no uncovered image areas.

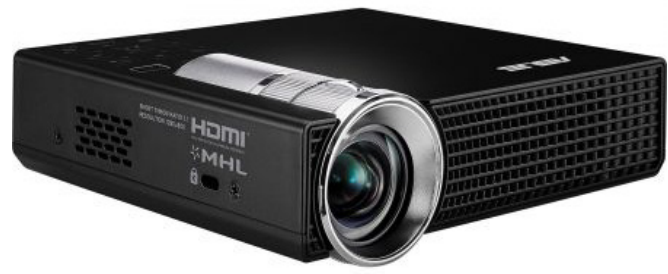

Figure 4 Short throw video projector [3]

The hardware part comprises a small form factor (SFF) computer type or a laptop with at least average hardware features to help the system run the software at a speed and optimum refresh rate. Obviously, all of these elements are brought together and connected over the sandbox, whose dimension is correlated with the video projector performance related to the diagonal dimensions of the formed image. The high price of this video projector recommends building the sandbox according to its performance, after its purchase. An important element is the arrangement of the kinect sensor and the video projector that are located at the top 
fixed on a variable length arm. If the performance of the videoprojector is higher than the minimum, then the size of the sand box may be higher, which is good for increasing the number of people in the field analysis.

\section{Results}

A schematic diagram of this complete is shown in the figure 5 .

The positioning of the video projector and motion sensor are essential because they must be placed in positions that cover the entire surface of the sandbox. The motion sensor is positioned vertically at the intersection of sandcase diagonals so that infrared camera distances at the corners of the box are equal so that no distance measurement errors are detected. Like any application that uses this type of sensor, it is important to calibrate the infrared camera for a correct distance measurement. The position of the video projector can easily be deviated from the vertical of the case because its lens may be oriented from the menu but will have to cover the entire surface covered by the sand with the image. The amount of sand placed inside the case can be variable but for the beginning it is approximated to $100 \mathrm{~kg}$ but it is possible to increase to $125 \mathrm{~kg}$ when the plotting terrain is medium or heavily rugged (mountainous).

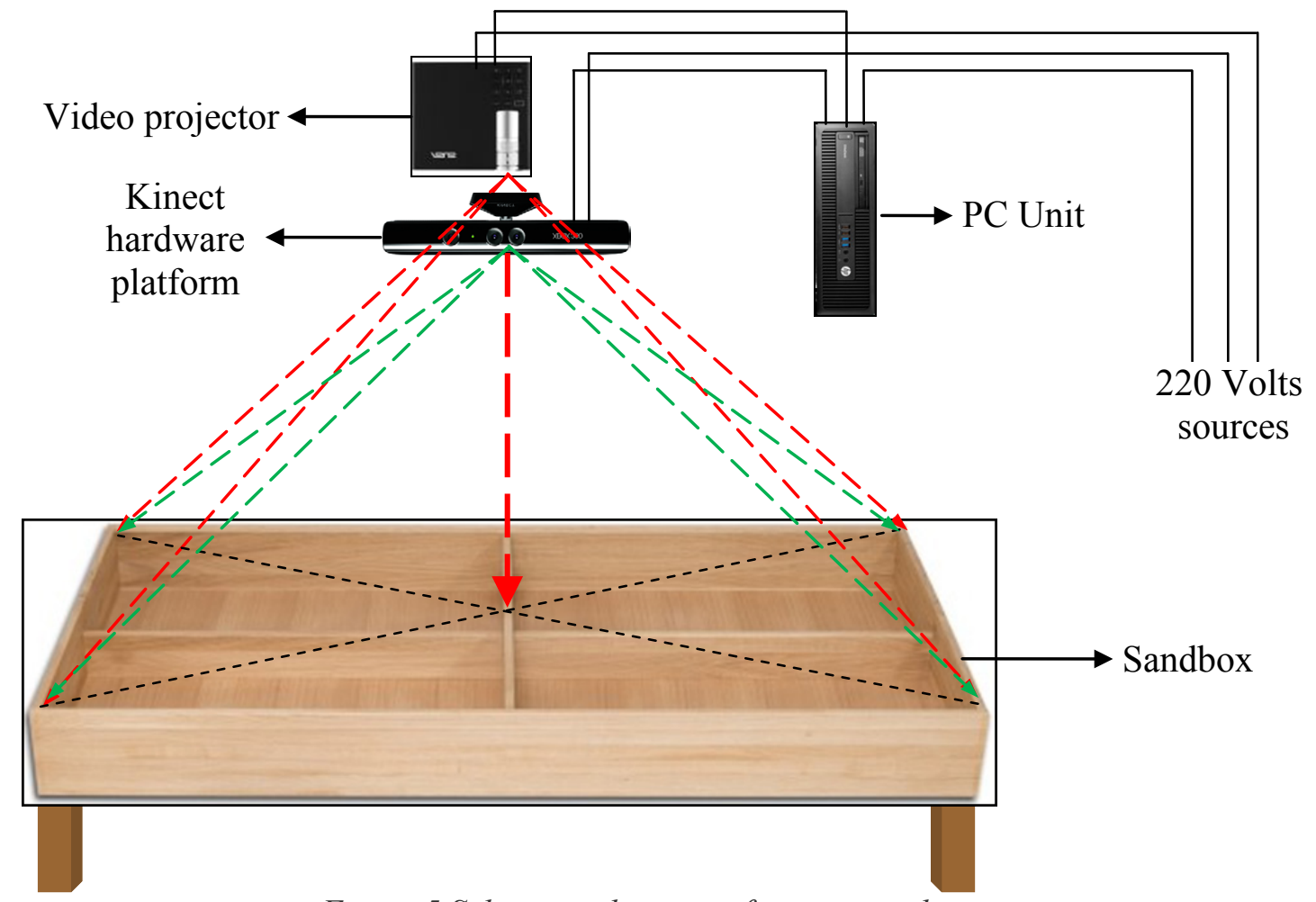

Figure 5 Schematic diagram of component layout

\section{Conclusions}

The realization of this virtual sandbox brings benefits to users in terms of the obviously better image quality and the easiness which such analysis is being prepared. Estimated costs for implementation are obviously higher in this first stage because hardware components are competitive, so expensive, but the medium and long term benefits in terms of efficiency are positive. Another major advantage is the short time in which the analysed area of land can be reshaped compared to a classical sandbox at which the zero land plot requires time, different material resources from a plot of land in another plot of land and the human contribution, which is even more important 
as the analyzed area is more complex and richer in details of the planimetry.

The land has always been an element that has had a major, sometimes even decisive, influence on military actions, because its knowledge and interpretation leads to the use of the maximum potential of fighting power of its own forces. A terrain analysis always begins with the analysis of existing and predicted conditions and continues with the influence and opportunities that it offers to forces to gain an important advantage in battle.

\section{References}

[1] http://www.danieli-ltd.com/productsimages/J894707.jpg

[2] http://091labs.com/wp-content/uploads/2010/11/kinect-20100613095507381.jpg

[3] https://www.badabum.ro/videoproiectoare/asus/43682-proiector-portabil-asus-p2e-dlpwxga-1280x800-350-lumeni-3500-1/ 